\title{
A Simulation Study of the Formation of Large-Scale Cyclonic and Anticyclonic Vortices in the Vicinity of the Intertropical Convergence Zone
}

\author{
Igor V. Mingalev, ${ }^{1}$ Natalia M. Astafieva, ${ }^{2}$ Konstantin G. Orlov, ${ }^{1}$ Victor S. Mingalev, \\ Oleg V. Mingalev, ${ }^{1}$ and Valery M. Chechetkin ${ }^{3}$ \\ ${ }^{1}$ Polar Geophysical Institute, Kola Scientific Center of the Russian Academy of Sciences, Murmansk Region, Apatity 184209, Russia \\ ${ }^{2}$ Space Research Institute of the Russian Academy of Sciences, Moscow 117997, Russia \\ ${ }^{3}$ Keldysh Institute of Applied Mathematics of the Russian Academy of Sciences, Moscow 125047, Russia
}

Correspondence should be addressed to Victor S. Mingalev; mingalev@pgia.ru

Received 31 January 2013; Accepted 26 February 2013

Academic Editors: A. De Santis, M. Ernesto, and A. Streltsov

Copyright ( 2013 Igor V. Mingalev et al. This is an open access article distributed under the Creative Commons Attribution License, which permits unrestricted use, distribution, and reproduction in any medium, provided the original work is properly cited.

\begin{abstract}
A regional nonhydrostatic mathematical model of the wind system of the lower atmosphere, developed recently in the Polar Geophysical Institute, is utilized to investigate the initial stage of the origin of large-scale vortices at tropical latitudes. The model produces three-dimensional distributions of the atmospheric parameters in the height range from 0 to $15 \mathrm{~km}$ over a limited region of the Earth's surface. Time-dependent modeling is performed for the cases when, at the initial moment, the simulation domain is intersected by the intertropical convergence zone (ITCZ). Calculations are made for various cases in which the initial forms of the intertropical convergence zone are different and contained convexities with distinct shapes, which are consistent with the results of satellite microwave monitoring of the Earth's atmosphere. The results of modeling indicate that the origin of convexities in the form of the intertropical convergence zone, having distinct configurations, can lead to the formation of different large-scale vortices, in particular, a cyclonic vortex, a pair of cyclonic-anticyclonic vortices, and a pair of cyclonic vortices, during a period not longer than three days. The radii of these large-scale vortices are about $400-600 \mathrm{~km}$. The horizontal wind velocity in these vortices can achieve values of $15-20 \mathrm{~m} / \mathrm{s}$ in the course of time.
\end{abstract}

\section{Introduction}

It is known that severe tropical cyclonic storms and hurricanes can cause tremendous damage and numerous fatalities. Therefore, prediction of tropical cyclone formation is a very important problem. Despite considerable efforts, the physical theory of tropical cyclone formation is still far from completion, even if some aspects of tropical cyclogenesis are commonly understood, in particular, in the late stages of formation as well as in a fully developed stage (see [1-4] and references therein). For tropical storm forecasting, it is necessary to investigate the pregenesis evolution of tropical cyclone. This investigation assumes that physical aspects of tropical cyclone formation must to be studied.
To investigate physical mechanisms responsible for the tropical cyclone formation, mathematical models may be utilized. Most of numerical studies of tropical cyclogenesis explore how a tropical cyclone forms from vortices, those are precursors to tropical cyclones, or from other preexisting large-scale disturbances of the troposphere [5-12].

In the Polar Geophysical Institute (PGI), the nonhydrostatic model of the global wind system in the Earth's atmosphere has been developed not long ago $[13,14]$. This model enables to calculate three-dimensional global distributions of the zonal, meridional, and vertical components of the wind at levels of the troposphere, stratosphere, mesosphere, and lower thermosphere, with whatever restrictions on the vertical transport of the atmospheric gas being absent. This 
model has been utilized in order to investigate numerically how the horizontal nonuniformity of the atmospheric gas temperature affects the formation of the middle atmosphere circulation for conditions corresponding to different seasons [13-16] and how solar activity affects the formation of the large-scale global circulation of the mesosphere and lower thermosphere [17].

Recently, a regional mathematical model of the wind system of the lower atmosphere has been developed in the Polar Geophysical Institute [18]. This model was applied to investigate the formation mechanisms of a large-scale vortex over a warm water band on the ocean surface. The results of modeling have allowed the authors to distinguish one of the formation mechanisms of moderate cyclones over the ocean [18].

Another formation mechanism of a cyclone was investigated, using this mathematical model, in the study by Belotserkovskii et al. [19]. In this study, it was shown that cyclones can appear in horizontally stratified shear flows of warm and wet air masses with a meridional direction of gradients of the wind velocity components as a result of small disturbances of pressure which can be produced by Rossby waves.

Also, this mathematical model has been applied to verify the hypothesis of the influence of the shape of the intertropical convergence zone (ITCZ) on the process of the formation of tropical cyclones. It was shown that the origin of a convexity in the configuration of the intertropical convergence zone can lead to the formation of a cyclonic vortex during the period of about one day. Its center is close to the southern edge of the initial intertropical convergence zone [20]. The results of mathematical modeling have indicated that the origin of a convexity of the intertropical convergence zone, having the specific forms, can lead to the formation of not only a single cyclonic vortex but also a pair of large-scale vortices [21-23].

The purpose of the present work is to continue these studies and to investigate numerically, applying the regional mathematical model of the wind system of the lower atmosphere developed in the PGI, the initial stage of the origin of large-scale vortices in the vicinity of the intertropical convergence zone. The applied model produces distributions of the lower atmosphere parameters in the limited threedimensional simulation domain. Time-dependent modeling is performed for various cases when, at the initial moment, the simulation domain is intersected by the intertropical convergence zones with different configurations. Calculations are made for some cases in which the initial forms of the intertropical convergence zone are different and contained convexities with distinct shapes.

\section{Mathematical Model}

In this study, the regional nonhydrostatic mathematical model of the wind system of the lower atmosphere, developed not long ago at the Polar Geophysical Institute, is applied. In this model, the atmospheric gas is considered as a mixture of air and water vapor, in which two types of precipitating water (namely, water microdrops and ice microparticles) can exist. The model is based on the numerical solution of the system of transport equations containing the equations of continuity for air and for the total water content in all phase states, momentum equations for the zonal, meridional, and vertical components of the air velocity, and energy equation. The characteristic feature of the model is that the vertical component of the air velocity is calculated without using the hydrostatic equation. Instead, the vertical component of the air velocity is obtained by means of a numerical solution of the appropriate momentum equation, with whatever simplifications of this equation being absent. In the momentum equations for all components of the air velocity, the effect of the turbulence on the mean flow is taken into account by using an empirical subgrid-scale parameterization similar to the global circulation model of the Earth's atmosphere developed earlier in the PGI $[13,14]$.

Thus, the utilized mathematical model is based on numerical solving of nonsimplified gas dynamic equations and produces three-dimensional time-dependent distributions of the wind components, temperature, air density, water vapor density, concentration of microdrops of water, and concentration of ice particles. The model takes into account heating/cooling of the air due to absorption/emission of infrared radiation, as well as due to phase transitions of water vapor to microdrops of water and ice particles, which play an important role in energetic balance. The finite-difference method and an explicit scheme are applied for solving the system of governing equations.

In the model calculations, the following variables are computed at each grid node: the temperature of the mixture of air and water vapor, $T$; densities of air and water vapor, $\rho_{a}$ and $\rho_{v}$, respectively; hydrodynamic velocity of the mixture (a $3 \mathrm{D}$ vector), $\vec{v}$; and the total mass of water microdrops and ice microparticles in a unit volume, $\rho_{w}$ and $\rho_{i}$, respectively. The governing equations in vectorial form can be written as follows:

$$
\begin{gathered}
\frac{\partial \rho_{a}}{\partial t}+\operatorname{div}\left(\rho_{a} \vec{v}\right)=0, \\
\frac{\partial\left(\rho_{v}+\rho_{w}+\rho_{i}\right)}{\partial t}+\operatorname{div}\left[\rho_{v} \vec{v}+\rho_{w}\left(\vec{v}+\vec{v}_{w}^{\text {prec }}\right)\right. \\
\left.+\rho_{i}\left(\vec{v}+\vec{v}_{i}^{\text {prec }}\right)\right]=0, \\
\frac{\partial\left(\rho_{\text {mix }} \vec{v}\right)}{\partial t}+\operatorname{div}\left(\rho_{\text {mix }} \vec{v} \otimes \vec{v}\right) \\
=-\nabla p+\operatorname{div} \vec{\tau}+\left(\rho_{\text {mix }}+\rho_{w}+\rho_{i}\right) \vec{F}, \\
\frac{\partial W}{\partial t}+\operatorname{div}\left[W_{\text {mix }} \vec{v}+W_{w}\left(\vec{v}+\vec{v}_{w}^{\text {prec }}\right)+W_{i}\left(\vec{v}+\vec{v}_{i}^{\text {prec }}\right)\right] \\
=\left[\rho_{\text {mix }} \vec{v}+\rho_{w}\left(\vec{v}+\vec{v}_{w}^{\text {prec }}\right)+\rho_{i}\left(\vec{v}+\vec{v}_{i}^{\text {prec }}\right), \vec{F}\right] \\
+\operatorname{div}(\vec{\tau} \cdot \vec{v}-p \vec{v}-\vec{j})+Q,
\end{gathered}
$$

where $\vec{v}_{w}^{\text {prec }}$ and $\vec{v}_{i}^{\text {prec }}$ are the precipitation velocities of water microdrops and ice microparticles, respectively, conditioned by the presence of an external force field and determined from 
the Stokes relation with Cunningham's correction; $\rho_{\text {mix }}=$ $\rho_{a}+\rho_{v} ; p$ is the pressure of the mixture defined as $p=$ $\left(\rho_{a} R_{a}+\rho_{v} R_{v}\right) T$, where $R_{a}$ and $R_{v}$ are the gas constants of air and water vapour, respectively; $\bar{\tau}$ is the extra stress tensor whose components are given by the rheological equation of state or the law of viscous friction which is the same as in the global circulation model of the Earth's atmosphere developed earlier [13, 14], with the effect of a small-scale turbulence, having the scales equal and less than the steps of finite-difference approximations, on the mean flow having been taken into account; $\vec{F}$ is the acceleration due to an external force field, which consists of the gravity acceleration, Coriolis acceleration, and acceleration of translation, that can be written in the form:

$$
\vec{F}=\vec{g}-2 \vec{\Omega} \times \vec{v}-\vec{\Omega} \times(\vec{\Omega} \times \vec{r}),
$$

where $\vec{g}$ is the acceleration due to gravity, $\vec{\Omega}$ is the Earth's angular velocity, and $\vec{r}$ is a radius vector from the center of the Earth to the point where the equation is applied. The following notations are used in (2):

$$
\begin{gathered}
W_{i}=\rho_{i}\left[\frac{1}{2}\left(\vec{v}+\vec{v}_{i}^{\text {prec }}\right)^{2}+C_{i} T\right], \\
W_{w}=\rho_{w}\left[\frac{1}{2}\left(\vec{v}+\vec{v}_{w}^{\text {prec }}\right)^{2}+C_{w}\left(T-T_{0}\right)+q_{\text {mel }}+C_{i} T_{0}\right], \\
W=W_{\text {mix }}+W_{w}+W_{i},
\end{gathered}
$$

where $C_{i}$ and $C_{w}$ are the specific heat capacities of ice and water, respectively, which are assumed constant; $T_{0}$ is the freezing temperature of water; $q_{\mathrm{mel}}$ is the specific heat of ice melting at $T=T_{0} ; q_{\mathrm{ev}}^{0}$ is the specific heat of water evaporation at $T=T_{0}$; also, the vector of heat flux, $\vec{j}$, is given by the well-known formula, $\vec{j}=-\bar{\lambda} \nabla T$, where $\bar{\lambda}$ is the symmetric tensor of thermal conductivity coefficients; and $Q$ is the rate of change of energy in a unit volume due to absorption/emission of infrared radiation. Concrete expressions of the model parameters, those appear in (1)-(2), may be found in the studies of Belotserkovskii et al. [18, 19].

It can be noticed that the model assumes that the water microdrops can exist only in the presence of saturated water vapor on condition that $T \geq T_{0}$, while the ice microparticles can exist only in the presence of saturated water vapor on condition that $T \leq T_{0}$. At $T=T_{0}$, the temperature of the matter cannot increase until all ice microparticles melt, and it cannot decrease until all water microdrops freeze.

The three-dimensional simulation domain of the model is a part of a spherical layer stretching from land and ocean surface up to the altitude of $15 \mathrm{~km}$ over a limited region of the Earth's surface. The dimensions of this region in longitudinal and latitudinal directions are $32^{\circ}$ and $25^{\circ}$, respectively. The finite-difference method and explicit scheme are applied for solving the system of governing equations. The calculated parameters are determined on a uniform grid. The latitude and longitude steps are equal to $0.08^{\circ}$, and height step is equal to $200 \mathrm{~m}$. Complete details of the utilized finite-difference method and numerical schemes have been presented in the paper of Mingalev et al. [24]. More complete details of the applied regional mathematical model may be found in the studies of Belotserkovskii et al. [18, 19] and Mingalev et al. [23].

\section{Presentation and Discussion of Results}

Modern scientific facility does not allow somebody to measure detailed three-dimensional fields of thermodynamical and gas dynamical parameters of the lower atmosphere with sufficient accuracy to understand the intrinsic nature of tropical cyclone genesis. However, for a better conceptual understanding of tropical cyclogenesis, satellite microwave observations may be applied, in particular, the results of satellite microwave monitoring of the Earth's atmosphere, collected at the Space Research Institute of the Russian Academy of Sciences (RAS) and included in the electronic data base "GLOBAL-Field" (http://www.iki.rssi.ru/asp/). This data base contains global radiothermal fields of the Earth at the frequencies containing the information about a moisture and water integral content distribution in the troposphere. The data were obtained from the spacecraft mission, DMSP (Defense Meteorological Satellite Program), with the help of the instrument, SSM/I (Special Sensor Microwave/ Imager).

As examples of data from the electronic collection "GLOBAL-Field," fragments of global radiothermal fields over the Atlantic Ocean are presented in Figures 1 and 2. In these Figures, the various colors of the image show the distribution of radiobrightness temperature of surface of ocean and land. It is essential that this temperature is consistent with the moisture and water integral content distribution in the troposphere. From Figures 1 and 2, one can see that the Atlantic Ocean is intersected by a red band stretched from Africa to South America. This band represents an intertropical convergence zone. It can be noticed that an intertropical convergence zone is similar to a band, in which zonal westward flow of air predominates, with the air velocity being enhanced. The width of the intertropical convergence zone can achieve a value of some hundreds of kilometers.

Studying numerous results of satellite microwave monitoring of the Earth's atmosphere, included in the electronic data base "GLOBAL-Field," we noticed that the form of an intertropical convergence zone may be different and, sometimes, can contain convexities with distinct shapes. Just such examples are presented in Figures 1 and 2, where the convexities are indicated by black pointers. As a consequence of our studies of results of satellite microwave monitoring of the Earth's atmosphere, we have advanced a hypothesis of the important role of the shape of the intertropical convergence zone in the process of the formation of tropical cyclones.

To verify the advanced hypothesis, we have decided to make calculations with the help of the regional nonhydrostatic mathematical model of the wind system of the lower atmosphere, described above, with the south boundary of the simulation domain having been disposed in the vicinity of the equator. It was supposed that the simulation domain is located between $5^{\circ} \mathrm{S}$ and $20^{\circ} \mathrm{N}$ and situated over the Atlantic Ocean. Simulations were performed for the cases 


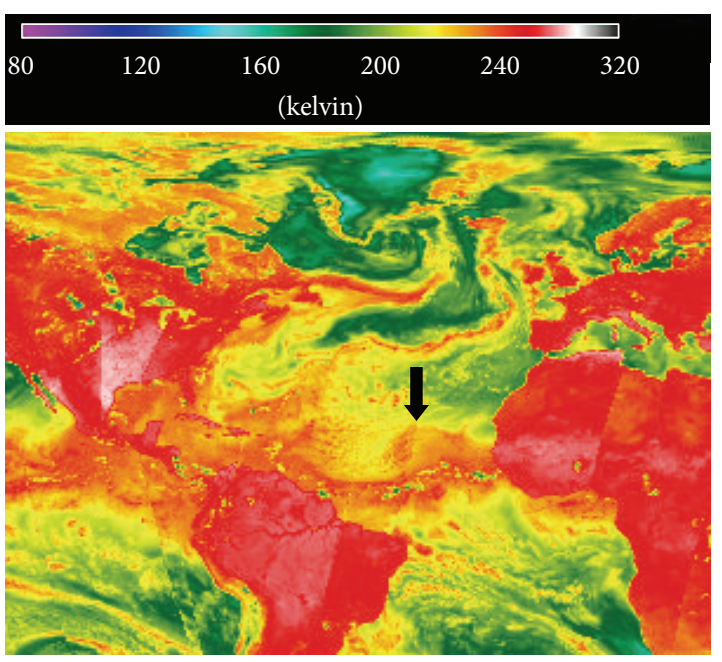

FIGURE 1: The fragment of global radiothermal field, derived from the results of satellite microwave monitoring of the Earth's atmosphere, obtained on June 29, 2005.

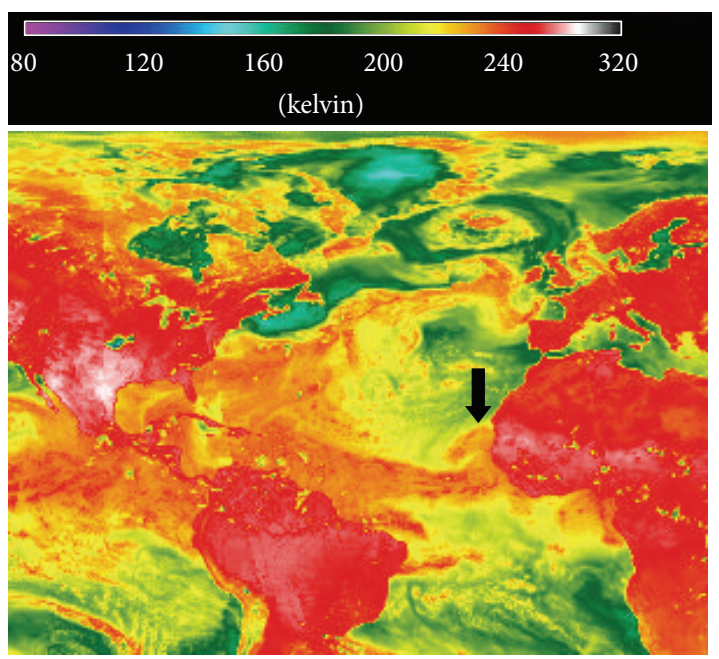

FIGURE 2: The same as in Figure 1, but obtained on July 3, 2005.

when the simulation domain is intersected by an intertropical convergence zone in the westeast direction.

From observation, it is known that, in an intertropical convergence zone, a zonal flow of air is westward, with the horizontal wind velocity being enhanced inside an intertropical convergence zone. A meridional wind velocity directs towards the centerline of an intertropical convergence zone at levels less than approximately $3 \mathrm{~km}$ and directs from the centerline of an intertropical convergence zone at levels higher than approximately $3 \mathrm{~km}$. A vertical wind velocity in an intertropical convergence zone is upward. Therefore, an intertropical convergence zone may be considered as a fluid stream, having enhanced zonal velocities, in the ambient atmospheric gas. In our calculations, we define the initial and boundary conditions as consistent with the situation when the intertropical convergence zone intersects the simulation domain in the westeast direction. Calculations are made for various cases in which the initial forms of the intertropical convergence zone are different and contained convexities with distinct shapes, which are consistent with observational data, included in the electronic collection "GLOBAL-Field." Since the obtained results are different, it is convenient to present them separately.

3.1. The Formation of a Cyclonic Vortex. Initially, let us consider the first case when, at the initial moment, the intertropical convergence zone contains a convexity in the north direction, with the deviation achieving a value of a few hundreds of kilometers. The initial form of the intertropical convergence zone may be easy seen from Figure 3(a), where it is like a light curved band. It is essential to note that, in the considered first case, the west crook of the convexity is sharp while the east crook of the convexity is gently sloping, with the west and east ends of the convexity being at the same latitudes.

The time evolution of model parameters was numerically simulated using the mathematical model during the period of about one day. The results of time-dependent modeling are partly shown in Figure 3. As can be seen from this figure, in the course of time, the initial distribution of horizontal component of the air velocity was considerably transformed. A cyclonic vortex flow arose whose center is close to the southern edge of the initial intertropical convergence zone. The horizontal wind velocity in this cyclonic vortex achieved a value of $20 \mathrm{~m} / \mathrm{s}$ during the period of twenty-seven hours. The radius of this large-scale cyclonic vortex is about $600 \mathrm{~km}$.

In addition, we made simulations for the second and third cases when, at the initial moment, the intertropical convergence zone has different configurations. For both cases, the initial forms of the intertropical convergence zone contained the convexities analogous to the convexity of the first case. However, for the second case, the east end of the convexity is situated at more northern latitudes than the west end of the convexity (see Figure 4(a)). On the contrary, for the third case, the east end of the convexity is situated at more southern latitudes than the west end of the convexity (see Figure 5(a)). The results of time-dependent modeling for the second and third cases of the initial configurations of the intertropical convergence zone are partly shown in Figures 4 and 5. As can be seen from these figures, in the course of time, cyclonic vortex flows arose whose centers are close to the southern edge of the initial intertropical convergence zone. These vortices are analogous to those obtained for the first case.

The simulation results indicate that physical reason of the formation of the calculated tropical cyclonic vortices is the origin of a convexity in the configuration of the intertropical convergence zone. As a rule, such convexities are observed during the periods of rebuilding of the global circulation of the atmosphere. The origin of a convexity of the intertropical convergence zone leads to the beginning of an instability of stream air flow. As a consequence, a large-scale vortex flow arises in the lower atmosphere, with its center being close to the southern edge of the initial intertropical convergence zone. When the mixture of air and water vapor moves to 


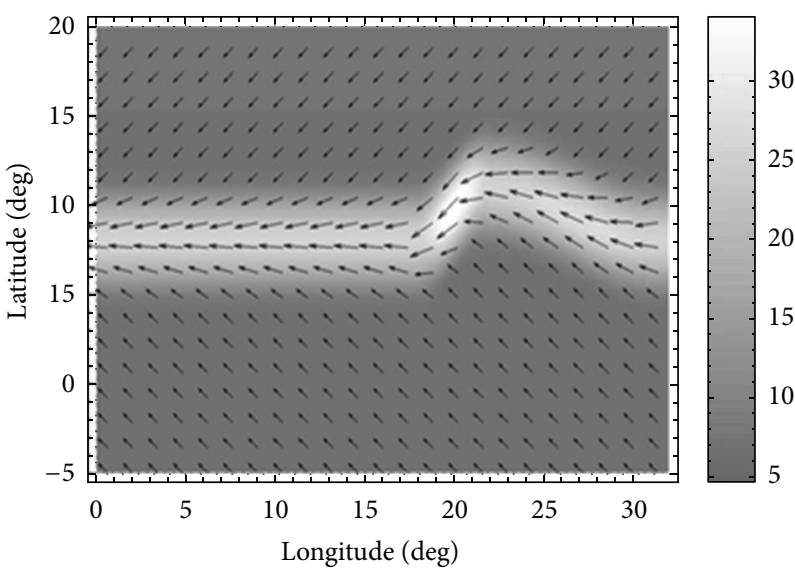

(a)

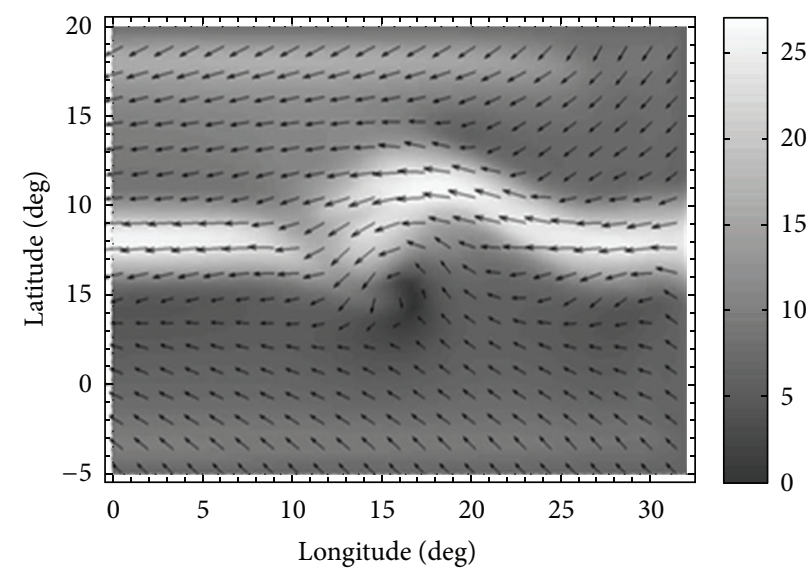

(b)

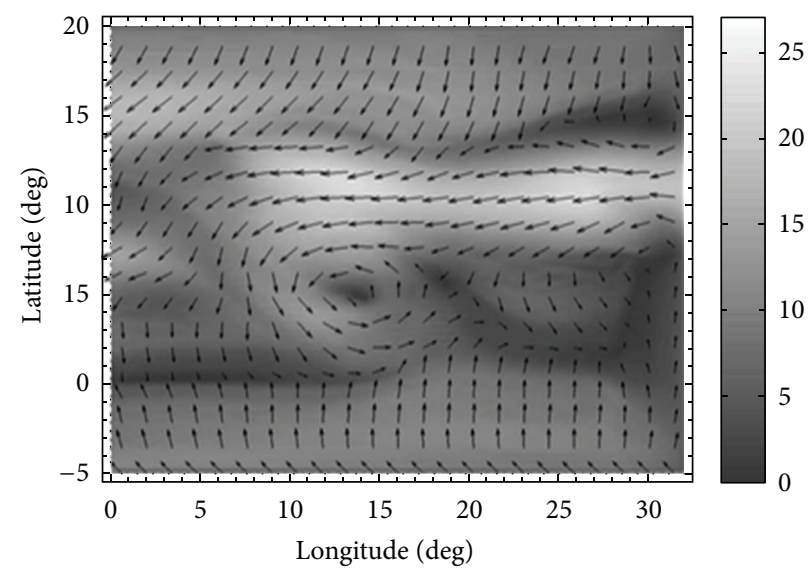

(c)

FIGURE 3: The distributions of horizontal component of the air velocity at the altitude of $600 \mathrm{~m}$, assigned at the initial moment (a), computed 12 hours after the beginning of calculations (b), and computed 27 hours after the beginning of calculations (c). The results are obtained for the first initial configuration of the intertropical convergence zone. The degree of shadowing of the figures indicates the module of the velocity in $\mathrm{m} / \mathrm{s}$.

higher altitudes, where temperature is lower, energy, emitted due to phase transitions of water vapor to microdrops of water and ice particles, transforms into kinetic energy of the air flow, with the horizontal wind velocity increasing in formed cyclonic vortex. It should be emphasized that the initial degree of saturation of air by water vapor plays an important role in the formation of the calculated tropical cyclonic vortices.

\subsection{The Formation of a Pair of Cyclonic-Anticyclonic Vortices.} In the previous subsection, we have considered three cases of the initial configuration of the intertropical convergence zone containing the convexity in the north direction, with the east end of the convexity located at different latitudes. The common feature of these initial configurations is that the west crook of the convexity is sharp while the east crook of the convexity is gently sloping. In the present subsection, we consider cases when both west and east crooks of the convexity are sharp and located at the same latitudes at the initial moment.

Initially, let us consider the fourth case when, at the initial moment, the intertropical convergence zone contains a convexity in the north direction. The initial form of the intertropical convergence zone may be easily seen from Figure 6(a), where it is like a light curved band. The time evolution of model parameters was numerically simulated using the mathematical model during the period of about one day. The results of time-dependent modeling are partly shown in Figure 6. As can be seen from this figure, in the course of time, the initial distribution of horizontal component of the air velocity was considerably transformed. A pair of cyclonic-anticyclonic vortices arose in the vicinity of the initial intertropical convergence zone. A cyclonic vortex arose whose center is close to the southern edge of the initial intertropical convergence zone and an anticyclonic vortex arose whose center is close to the northern edge of the initial intertropical convergence zone. The horizontal wind velocity in these vortices achieved a value of $20 \mathrm{~m} / \mathrm{s}$ during the period 


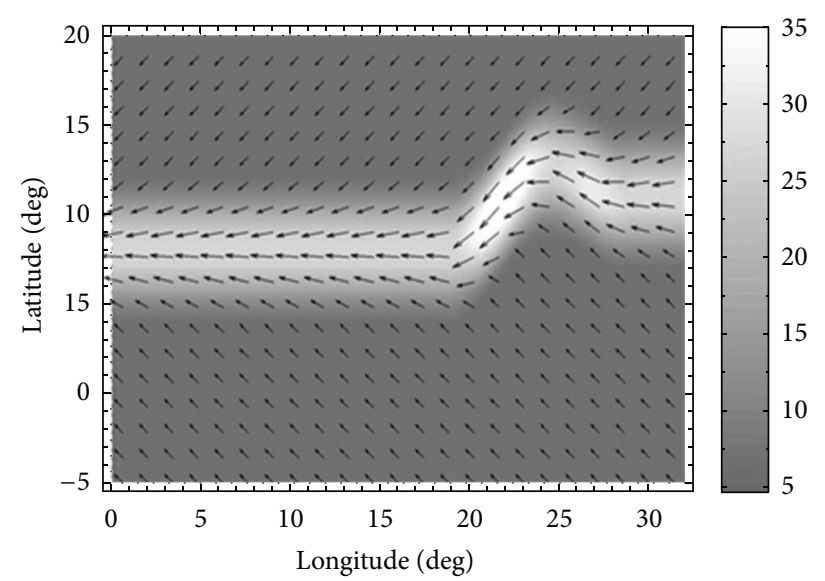

(a)

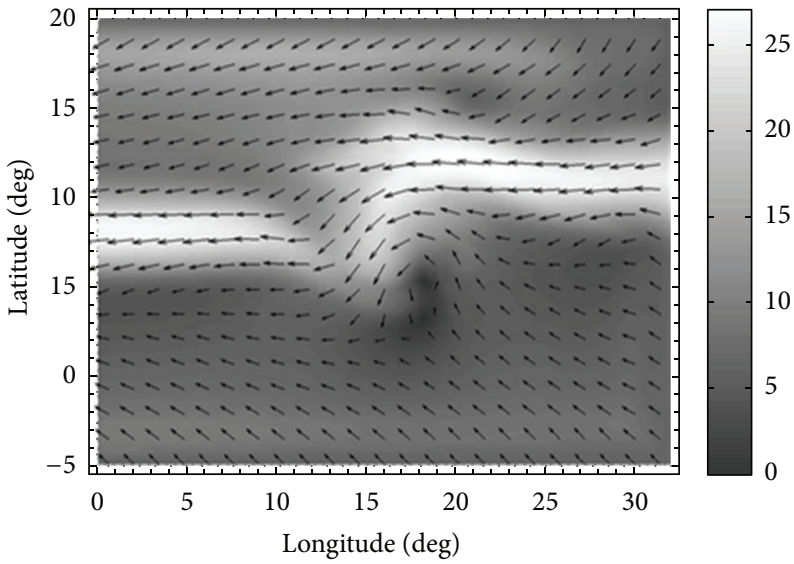

(b)

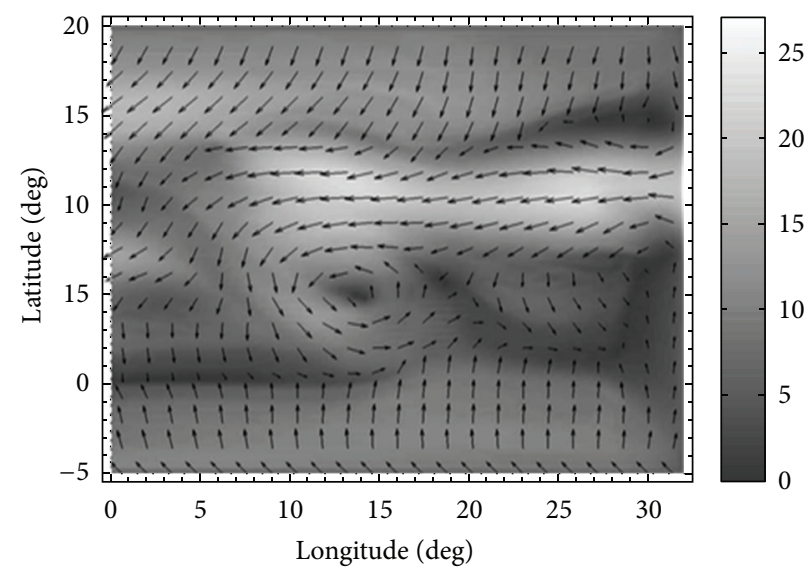

(c)

Figure 4: The same as in Figure 3, but obtained for the second initial configuration of the intertropical convergence zone. The degree of shadowing of the figures indicates the module of the velocity in $\mathrm{m} / \mathrm{s}$.

of twenty-seven hours. The radii of these large-scale vortices are about $400 \mathrm{~km}$.

It should be emphasized that the results of satellite monitoring of the Earth's atmosphere often indicated a simultaneous origin of a cyclone-anticyclone pair. Naturally, a formation of a single cyclone or a single anticyclone was observed by satellites repeatedly. It can be noticed that, according to observations, an initially originated single cyclonic or anticyclonic vortex as well as one of vortices, belonging to a cyclone-anticyclone pair, as well as both vortices, belonging to a cyclone-anticyclone pair, sometimes, can be attenuated in the course of time and will not achieve a status of the long-live large-scale atmospheric vortices.

Let us consider the fifth case when, at the initial moment, the intertropical convergence zone contains a convexity, analogous to the convexity of the fourth case, but deviated in the south direction. The initial form of the intertropical convergence zone may be easy seen from Figure 7(a), where it is like a light curved band. The results of time-dependent modeling for the fifth case of the initial configurations of the intertropical convergence zone are partly shown in Figure 7. As can be seen from this figure, in the course of time, a pair of cyclonic-anticyclonic vortices arose in the vicinity of the initial intertropical convergence zone. These vortices are analogous to those obtained for the fourth case.

The results of simulation indicate that the physical reason of the formation of the calculated pair of cyclonicanticyclonic vortices is the origin of a convexity in the configuration of the intertropical convergence zone, having the specific forms. The origin of a convexity of the intertropical convergence zone leads to the beginning of an instability of stream air flow. As a consequence, a pair of cyclonicanticyclonic vortices arises in the lower atmosphere. In the course of time, the horizontal wind velocity in the vortices increases due to a growth of kinetic energy of the air flow because of a transformation of energy, emitted owing to phase transitions of water vapor to microdrops of water and ice particles, in the flow of air moving to higher altitudes, where temperature is lower, with the initial degree of saturation of air by water vapor playing an important role in the formation of the calculated vortices.

3.3. The Formation of a Pair of Tropical Cyclonic Vortices. In the previous subsections, at the initial moment, the horizontal 


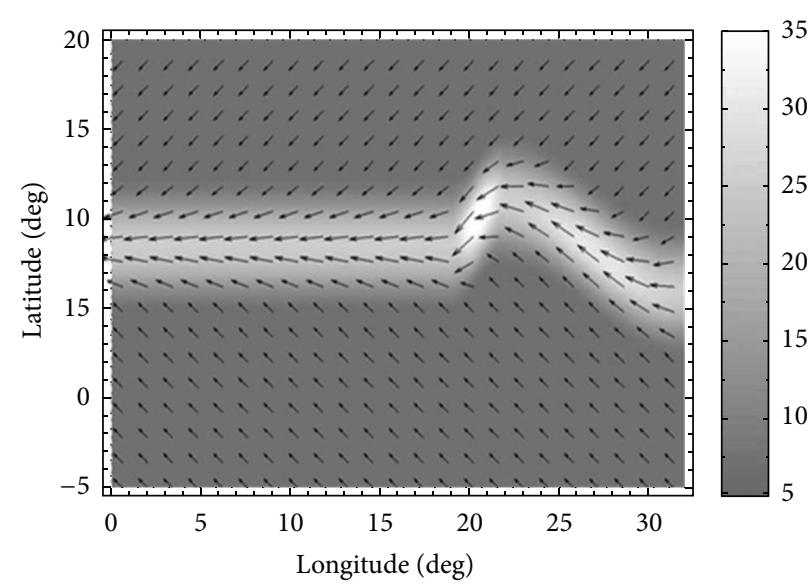

(a)

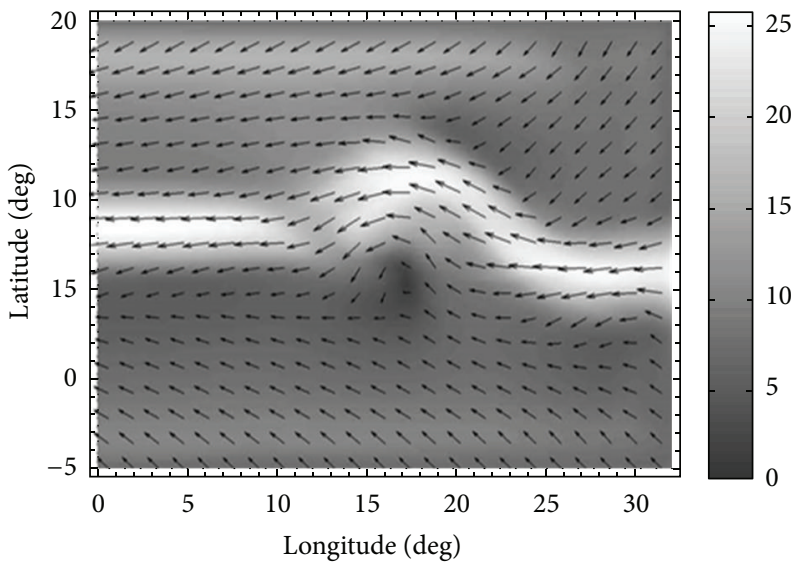

(b)

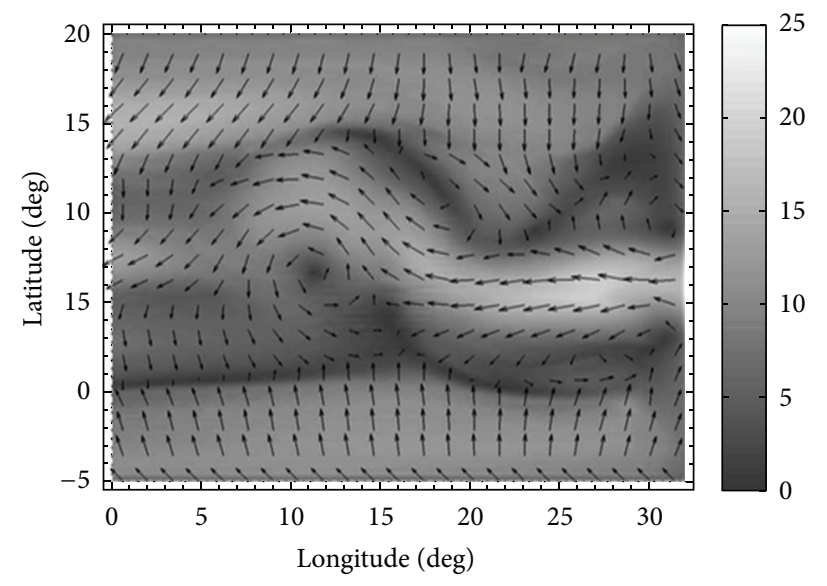

(c)

Figure 5: The same as in Figure 3, but obtained for the third initial configuration of the intertropical convergence zone. The degree of shadowing of the figures indicates the module of the velocity in $\mathrm{m} / \mathrm{s}$.

velocity fields were approximately symmetric relatively to the centerline of the intertropical convergence zone not only inside it but also beyond the intertropical convergence zone. In the present subsection, we consider a case when, at the initial moment, the horizontal velocity field is not symmetric relatively to the centerline of the intertropical convergence zone inside and beyond it. Moreover, in the present subsection, the dimension of the simulation domain in longitudinal direction is equal to $40^{\circ}$.

Let us consider the sixth case when, at the initial moment, the intertropical convergence zone contains a convexity in the north direction, with the zonal wind velocities at more northern latitudes relative to the centerline of the intertropical convergence zone being larger than at more southern latitudes relative to it. The initial form of the intertropical convergence zone may be easily seen from Figure 8(a), where it is like a light curved band. It is noted that, in the considered sixth case, the west crook of the convexity is sharp while the east crook of the convexity is gently sloping, with the east end of the convexity being $1^{\circ}$ southern than the west crook of it. The time evolution of model parameters was numerically simulated using the mathematical model during the period of about 3 days. The results of time-dependent modeling are partly shown in Figures 8 and 9. As can be seen from these figures, in the course of time, the initial distribution of horizontal component of the air velocity was considerably transformed. In a moment of 20 hours after the beginning of calculations, a cyclonic vortex flow arose whose center is close to the southern edge of the initial intertropical convergence zone. In a moment of 40 hours after the beginning of calculations, this cyclonic vortex has moved in the western direction for about 5 degrees. In a moment of 60 hours after the beginning of calculations, the rotational center of the formed cyclonic vortex has moved in the northwest direction for a distance of about $600 \mathrm{~km}$. Simultaneously, the second cyclonic vortex arose, with its center being close to the southern edge of the initial intertropical convergence zone.

Thus, in a moment of 70 hours after the beginning of calculations, a pair of cyclonic vortices arose in the vicinity of the intertropical convergence zone. The rotational centers of these cyclonic vortices are situated near the edges of the intertropical convergence zone. The center of the first cyclonic vortex is close to the northern edge while the center of the second cyclonic vortex is close to the southern edge 


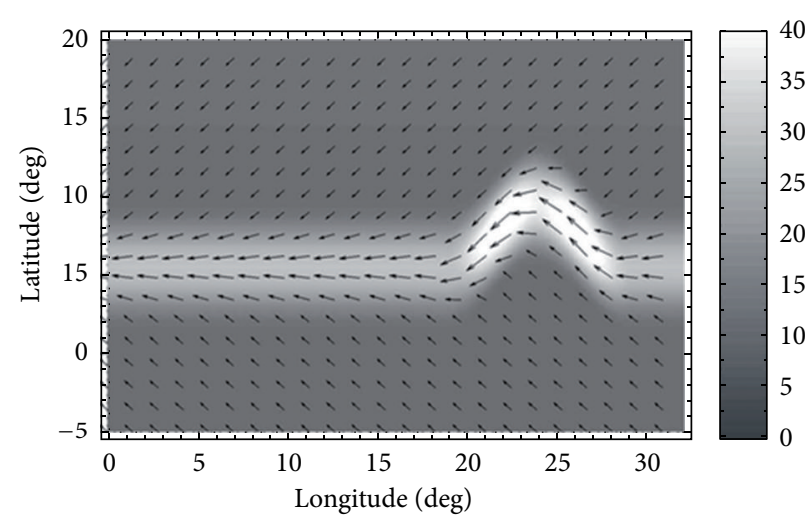

(a)

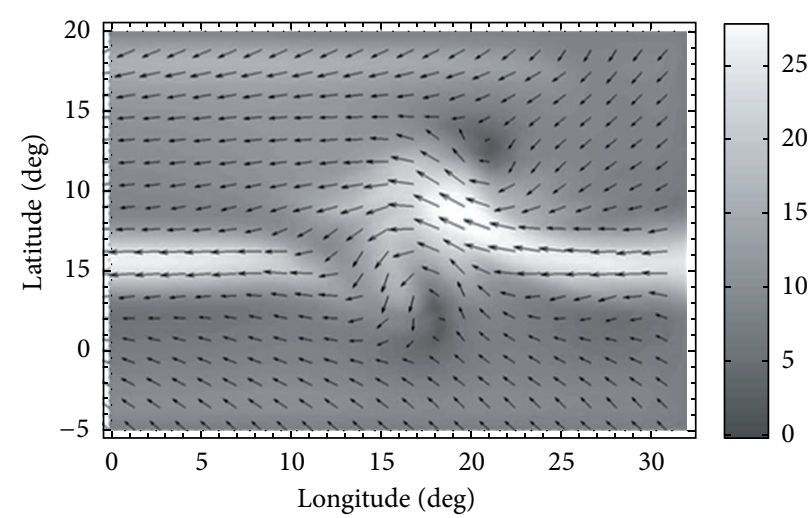

(b)

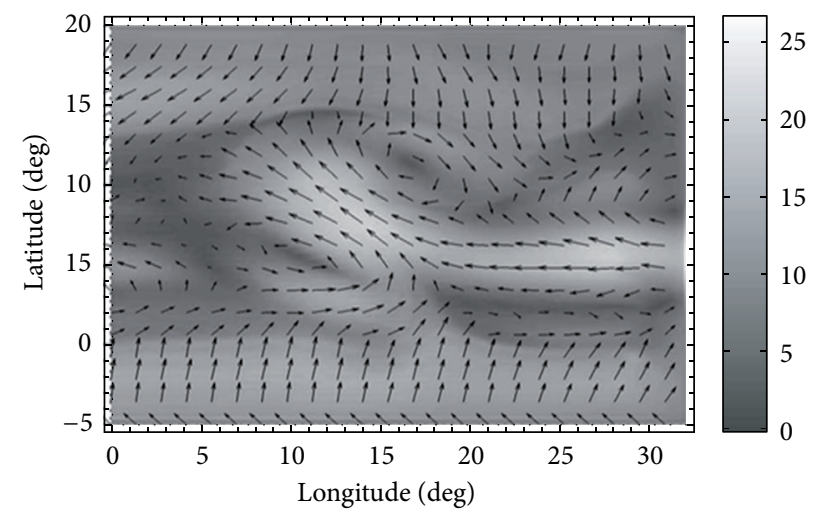

(c)

Figure 6: The distributions of horizontal component of the air velocity at the altitude of $600 \mathrm{~m}$, assigned at the initial moment (a), computed 12 hours after the beginning of calculations (b), and computed 27 hours after the beginning of calculations (c). The results are obtained for the fourth initial configuration of the intertropical convergence zone. The degree of shadowing of the figures indicates the module of the velocity in $\mathrm{m} / \mathrm{s}$.

of the intertropical convergence zone. The horizontal wind velocity in these cyclonic vortices achieved values of $15-$ $20 \mathrm{~m} / \mathrm{s}$ during the period of seventy hours. The radii of these cyclonic vortices are about $600 \mathrm{~km}$.

It can be noticed that the results of observation of the Earth's atmosphere indicated a simultaneous origin of twin tropical cyclones sometimes [25].

The results of simulation indicate that a key factor in the modeled formation of twin tropical cyclonic vortices is the origin of a convexity in the configuration of the intertropical convergence zone, having the specific form, which is accompanied by nonsymmetric horizontal velocity field in the vicinity of this zone. The pointed out factors lead to the beginning of instability of stream air flow. As a consequence, a pair of cyclonic vortices arise in the lower atmosphere in the course of time. A transformation of energy, emitted due to phase transitions of water vapor to microdrops of water and ice particles in the mixture of air and water vapor moving upward, into kinetic energy of the air flow plays an important role in the increase of the horizontal wind velocity in the course of time, with the high initial degree of saturation of air by water vapor being an important factor.

\section{Summary and Concluding Remarks}

A number of earlier studies have shown that the intertropical convergence zone is sometimes observed to undulate and break down into a series of tropical disturbances $[3,26$, 27]. Some of these disturbances may develop into tropical cyclones, while others dissipate, and the intertropical convergence zone may reform in the original region. It has been proposed that the intertropical convergence zone may break down because of its heating-induced potential vorticity anomalies. Moreover, it has been proposed that the intertropical convergence zone breakdown results from a convectively modified form of combined barotropic and baroclinic instability of the mean flow [26]. Also, instability of the intertropical convergence zone has been studied in the works of Wang and Magnusdottir [28] and Nolan et al. [29]. The role of tropical waves in tropical cyclogenesis has been studied, too [30].

Nevertheless, in earlier studies, we have not found the idea that the transformation of the shape of the intertropical convergence zone can influence the process of the formation of tropical cyclones (with the exception of the studies by the authors of the present work). Although, the position and 


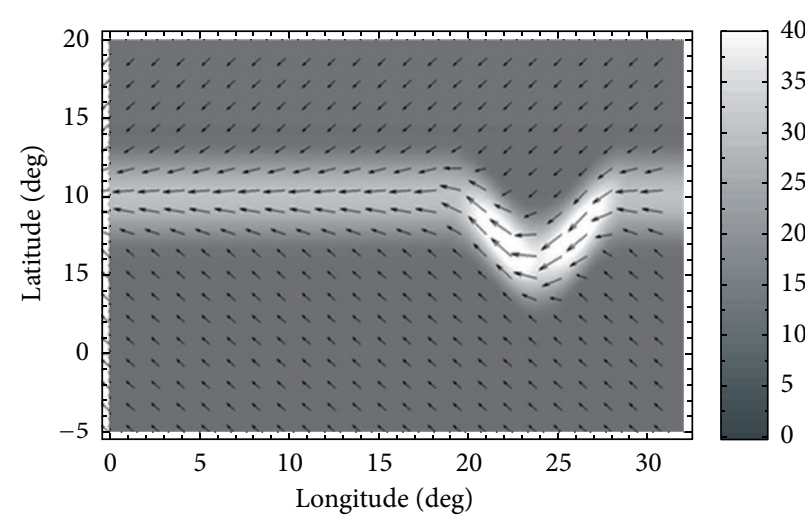

(a)

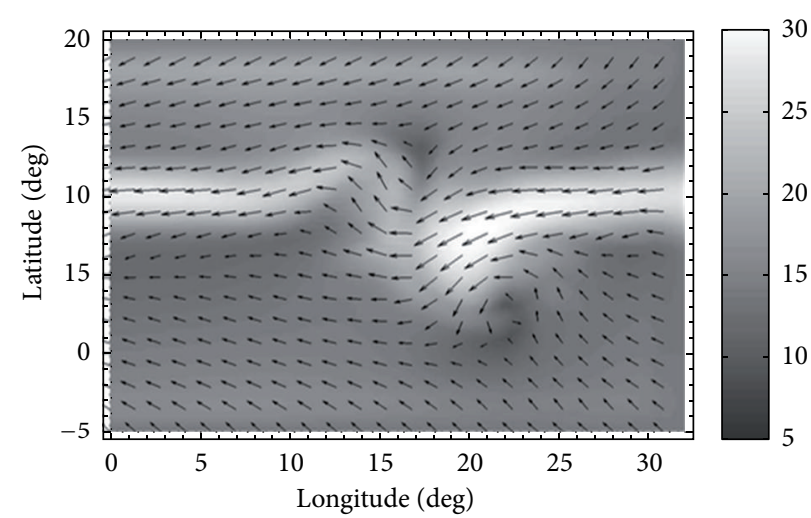

(b)

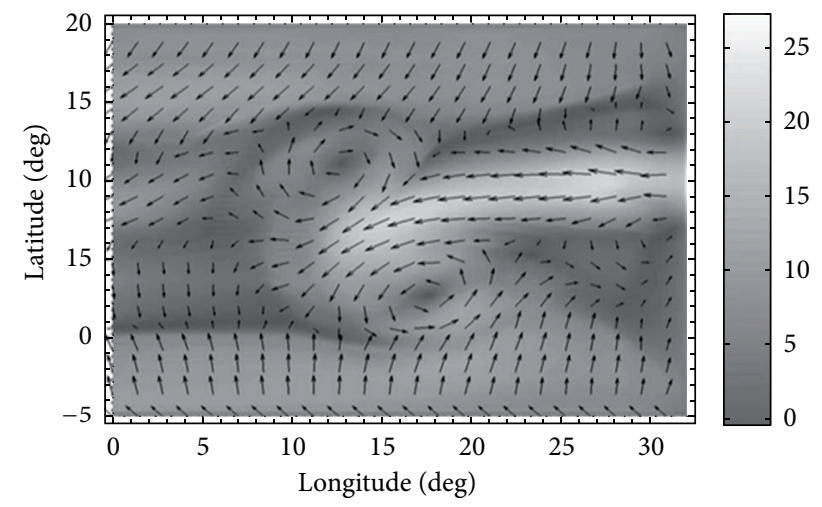

(c)

Figure 7: The same as in Figure 6, but obtained for the fifth initial configuration of the intertropical convergence zone. The degree of shadowing of the figures indicates the module of the velocity in $\mathrm{m} / \mathrm{s}$.

variability of the intertropical convergence zone have been studied in a number of earlier works [31-33]. It was found in these studies that the northsouth position of the intertropical convergence zone responds to changes in interhemispheric temperature contrast. An asymmetry in air-sea interactions can play an important role in forming the configuration of the intertropical convergence zone, too. In the present study, the influence of the disturbance of the configuration of the intertropical convergence zone on the process of the formation of tropical large-scale cyclonic and anticyclonic vortices was investigated numerically.

To make this investigation, the limited-area nonhydrostatic mathematical model of the wind system of the lower atmosphere, developed recently in the Polar Geophysical Institute, was utilized. The model produces threedimensional distributions of the atmospheric parameters in the height range from land and ocean surface up to the altitude of $15 \mathrm{~km}$ over a limited region of the Earth's surface. The time evolution of model parameters was numerically simulated using various variants of the initial and boundary conditions which were defined as consistent with the situation when the intertropical convergence zone intersects the simulation domain in the westeast direction. Calculations were made for various cases in which the initial forms of the intertropical convergence zone were different and contained convexities with distinct shapes, which were consistent with the results of satellite microwave monitoring of the Earth's atmosphere, included in the electron data base "GLOBALField," developed in the Space Research Institute of the RAS.

The results of modeling indicated that the origin of a convexity in the configuration of the intertropical convergence zone, having the latitudinal dimension of $800-1000 \mathrm{~km}$ and the deviation of some hundreds of kilometers either in north or south direction, can lead to the formation of tropical largescale vortices during the period of a few days. It was found that the initial shape of the convexity of the intertropical convergence zone plays a significant role in the process of the formation of tropical large-scale vortices.

Firstly, it was established the specific initial shapes of the intertropical convergence zone convexity, which bring forth a cyclonic vortex during the period of about one day. The common features of these initial shapes are that the convexities deviate in the north direction and that the west crook of the convexity is sharp while the east crook of the convexity is gently sloping. The east end of the convexity may be situated at more northern or at more southern latitudes than the west end of the convexity as well as at the same latitudes. The rotational center of the formed cyclonic vortex is close to the southern edge of the initial intertropical convergence zone. The cyclonic vortex has a horizontal extent 


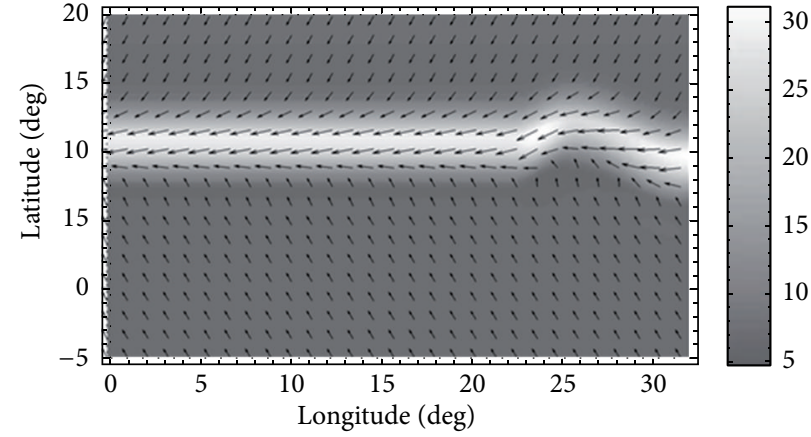

(a)

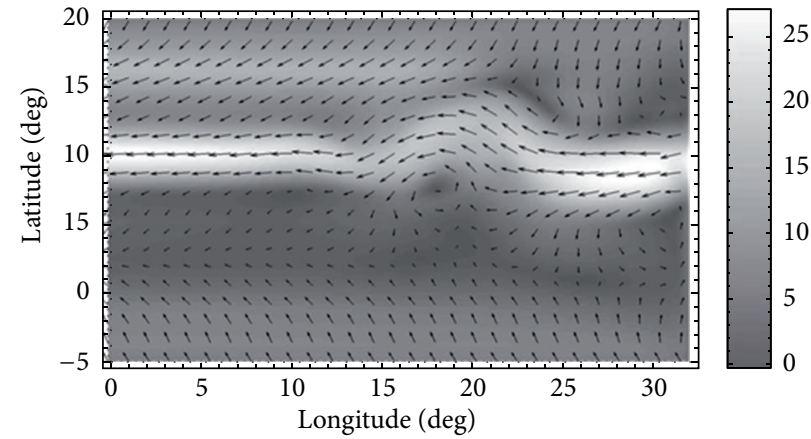

(b)

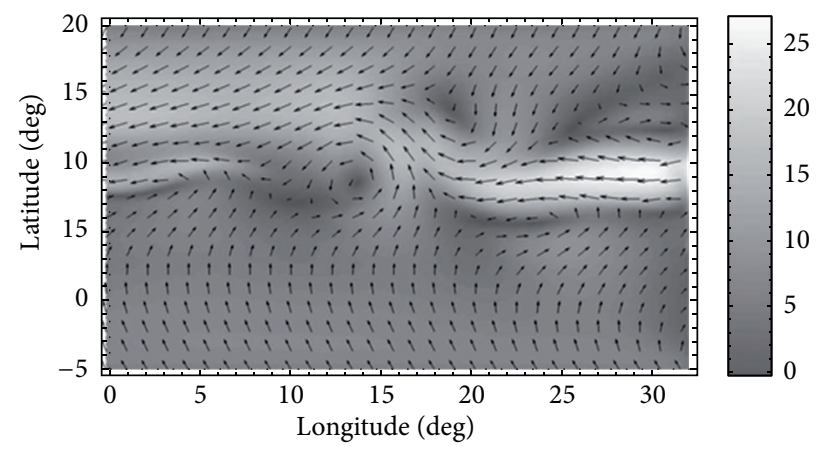

(c)

Figure 8: The distributions of horizontal component of the air velocity at the altitude of $600 \mathrm{~m}$, assigned at the initial moment (a), computed 20 hours after the beginning of calculations (b), and computed 40 hours after the beginning of calculations (c). The results are obtained for the sixth initial configuration of the intertropical convergence zone. The degree of shadowing of the figures indicates the module of the velocity in $\mathrm{m} / \mathrm{s}$.

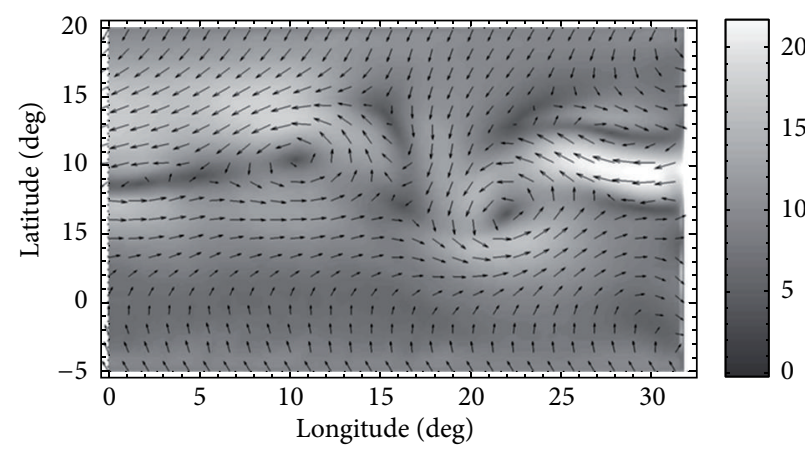

(a)

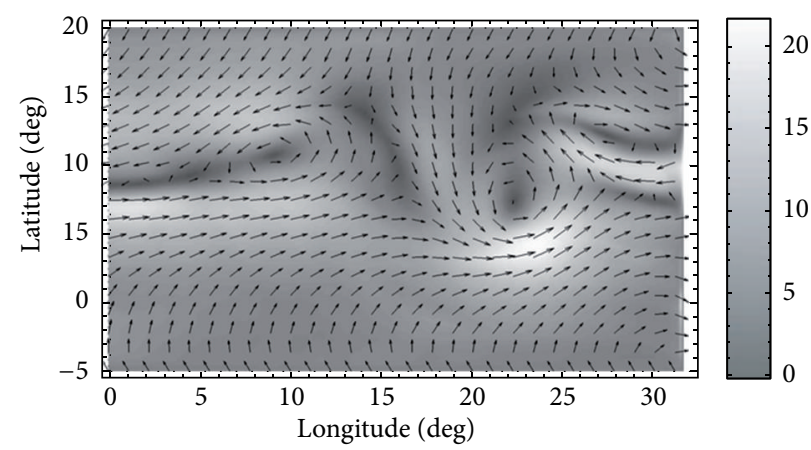

(b)

Figure 9: The same as in Figure 8, but computed 60 hours after the beginning of calculations (a) and 70 hours after the beginning of calculations (b). The results are obtained for the sixth initial configuration of the intertropical convergence zone. The degree of shadowing of the figures indicates the module of the velocity in $\mathrm{m} / \mathrm{s}$.

of about $600 \mathrm{~km}$. The horizontal wind velocity in this cyclonic vortex can achieve a value of $20 \mathrm{~m} / \mathrm{s}$ during the period of 27 hours.

Secondly, it was found the specific initial shapes of the intertropical convergence zone convexity, which bring forth a pair of cyclonic-anticyclonic vortices during the period of about one day. The common feature of these initial shapes is that both west and east crooks of the convexity are sharp at the initial moment. The convexity may be deviated either in the north direction or in the south direction, with the west and east ends of the convexity being at the same latitudes. The rotational center of the formed cyclonic vortex is close to the southern edge of the initial intertropical convergence zone while the rotational center of the formed anticyclonic vortex is close to the northern edge of the initial intertropical convergence zone to a moment of 27 hours after 
the beginning of calculations. The radii of these large-scale vortices are about $400 \mathrm{~km}$. The horizontal wind velocity in these vortices can achieve a value of $20 \mathrm{~m} / \mathrm{s}$ during the period of twenty-seven hours.

Thirdly, it was established the specific variant of the initial and boundary conditions which, during the period of about three days, bringing forth a pair of tropical cyclonic vortices. It was supposed that, at the initial moment, the intertropical convergence zone contains the convexity in the north direction; moreover, the zonal wind velocities at more northern latitudes relative to the centerline of the intertropical convergence zone are larger than those at more southern latitudes relative to it, with the west crook of the convexity being sharp while the east crook of the convexity being gently sloping. The cyclonic vortices were formed one after another in the course of time. The rotational center of the first cyclonic vortex is close to the northern edge while the center of the second cyclonic vortex is close to the southern edge of the initial intertropical convergence zone in a moment of 70 hours after the beginning of calculations. The radii of these cyclonic vortices are about $600 \mathrm{~km}$. The horizontal wind velocity in the cyclonic vortices achieved values of $15-20 \mathrm{~m} / \mathrm{s}$ during the period of about three days.

It should be emphasized that, according to observations, not each cyclonic or anticyclonic vortex, arisen in the lower atmosphere, has the potential to grow up to the long-live large-scale atmospheric vortex. It is known that, sometimes, a vortex, initially arisen in the lower atmosphere, can be attenuated in the course of time and will not achieve a status of the long-live large-scale atmospheric vortex. This peculiarity may take place for the large-scale vortices arisen in the calculations of the present study, which were limited by the time intervals not longer than three days. Unfortunately, more prolonged time intervals are impossible for the utilized mathematical model because of limited sizes of its simulation domain and owing to tendency of the modeled vortices to move and to abandon the simulation domain in the course of time.

The simulation results show that a key factor in the modeled formation of tropical large-scale vortices is the origin of a convexity in the configuration of the intertropical convergence zone. As a consequence, instability of stream air flow arises, presenting in the intertropical convergence zone. This instability leads to considerable transformation of the wind field. As a result, tropical large-scale vortices may be formed in the vicinity of the initial position of the intertropical convergence zone in the course of time. In addition to that, the initial intertropical convergence zone is broken down. Energy, emitted due to phase transitions of water vapor to microdrops of water and ice particles in the mixture of air and water vapor, moving to higher altitudes, where temperature is lower, transforms into kinetic energy of the air flow, with the horizontal wind velocity increasing in the formed vortices.

It may be expected that the simulation results of the present study will be useful for tropical cyclone forecasting. The origin of a convexity in the configuration of the intertropical convergence zone, which may be observed with the help of satellite microwave monitoring of the Earth's atmosphere, is a precursor of the formation of a tropical largescale vortex. This vortex either will grow up to the longlive large-scale atmospheric vortex, having the potential to transform to a tropical storm, or can be attenuated and vanish in the course of time. As a rule, intertropical convergence zone convexities originate during the periods of rebuilding of the global circulation of the atmosphere, in particular, from July to September over the Atlantic Ocean and Africa. Further transformation of the originated convexity of the intertropical convergence zone depends on its initial shape and on environmental conditions, in particular, on the degree of saturation of air by water vapor.

\section{Acknowledgment}

This work was partly supported by Grant no. 13-01-00063 from the Russian Foundation for Basic Research.

\section{References}

[1] K. A. Emanuel, "An air-sea interaction theory for tropical cyclones. Part I: steady-state maintenance," Journal of the Atmospheric Sciences, vol. 43, no. 6, pp. 585-605, 1986.

[2] M. T. Montgomery and B. F. Farrell, "Tropical cyclone formation,” Journal of the Atmospheric Sciences, vol. 50, no. 2, pp. 285310, 1993.

[3] C. Q. Kieu and D. L. Zhang, "Genesis of tropical storm eugene (2005) from merging vortices associated with ITCZ breakdown. Part I: observational and modeling analyses," Journal of the Atmospheric Sciences, vol. 65, no. 11, pp. 3419-3439, 2008.

[4] J. Mao and G. Wu, "Barotropic process contributing to the formation and growth of tropical cyclone Nargis," Advances in Atmospheric Sciences, vol. 28, no. 3, pp. 483-491, 2011.

[5] K. Ooyama, "Numerical simulation of the life cycle of tropical cyclones," Journal of the Atmospheric Sciences, vol. 26, no. 1, pp. 3-40, 1969.

[6] M. T. Montgomery and J. Enagonio, "Tropical cyclogenesis via convectively forced vortex Rossby waves in a three-dimensional quasigeostrophic model," Journal of the Atmospheric Sciences, vol. 55, no. 20, pp. 3176-3207, 1998.

[7] T. Li, X. Ge, B. Wang, and Y. Zhu, “Tropical cyclogenesis associated with Rossby wave energy dispersion of a preexisting typhoon. Part II: numerical simulations," Journal of the Atmospheric Sciences, vol. 63, no. 5, pp. 1390-1409, 2006.

[8] T. N. Venkatesh and J. Mathew, "A numerical study of the role of the vertical structure of vorticity during tropical cyclone genesis," Fluid Dynamics Research, vol. 42, no. 4, Article ID 045506, 2010.

[9] M. T. Montgomery, Z. Wang, and T. J. Dunkerton, "Coarse, intermediate and high resolution numerical simulations of the transition of a tropical wave critical layer to a tropical storm," Atmospheric Chemistry and Physics, vol. 10, no. 22, pp. 1080310827, 2010.

[10] Y. Xu, "The genesis of tropical cyclone Bilis (2000) associated with cross-equatorial surges," Advances in Atmospheric Sciences, vol. 28, no. 3, pp. 665-681, 2011.

[11] S. F. Abarca and K. L. Corbosiero, "Secondary eyewall formation in WRF simulations of Hurricanes Rita and Katrina (2005)," Geophysical Research Letters, vol. 38, no. 7, Article ID L07802, 2011. 
[12] K. A. Reed and C. Jablonowski, "Impact of physical parameterizations on idealized tropical cyclones in the Community Atmosphere Model," Geophysical Research Letters, vol. 38, no. 4, Article ID L04805, 2011.

[13] I. V. Mingalev and V. S. Mingalev, "The global circulation model of the lower and middle atmosphere of the Earth with a given temperature distribution," Mathematical Modeling, vol. 17, pp. 24-40, 2005 (Russian).

[14] I. V. Mingalev, V. S. Mingalev, and G. I. Mingaleva, "Numerical simulation of the global distributions of the horizontal and vertical wind in the middle atmosphere using a given neutral gas temperature field," Journal of Atmospheric and Solar-Terrestrial Physics, vol. 69, no. 4-5, pp. 552-568, 2007.

[15] I. V. Mingalev, O. V. Mingalev, and V. S. Mingalev, "Model simulation of the global circulation in the middle atmosphere for January conditions," Advances in Geosciences, vol. 15, pp. 1116, 2008.

[16] I. V. Mingalev, V. S. Mingalev, and G. I. Mingaleva, "Numerical simulation of the global neutral wind system of the Earth's middle atmosphere for different seasons," Atmosphere, vol. 3, no. 1, pp. 213-228, 2012.

[17] I. V. Mingalev and V. S. Mingalev, "Numerical modeling of the influence of solar activity on the global circulation in the Earth's mesosphere and lower thermosphere," International Journal of Geophysics, vol. 2012, Article ID 106035, 15 pages, 2012.

[18] O. M. Belotserkovskii, I. V. Mingalev, V. S. Mingalev, O. V. Mingalev, and A. M. Oparin, "Mechanism of the appearance of a large-scale vortex in the troposphere above a nonuniformly heated surface," Doklady Earth Sciences, vol. 411, no. 8, pp. 1284$1288,2006$.

[19] O. M. Belotserkovskii, I. V. Mingalev, V. S. Mingalev, O. V. Mingalev, A. M. Oparin, and V. M. Chechetkin, "Formation of large-scale vortices in shear flows of the lower atmosphere of the Earth in the region of tropical latitudes," Cosmic Research, vol. 47, no. 6, pp. 466-479, 2009.

[20] I. V. Mingalev, N. M. Astafieva, K. G. Orlov, V. S. Mingalev, and O. V. Mingalev, "Time-dependent modeling of the initial stage of the formation of cyclones in the intertropical convergence zone of the northern hemisphere," in Proceedings of the 33rd Annual Seminar on Physics of Auroral Phenomena, pp. 182-185, Polar Geophysical Institute, Apatity, Russia, 2011.

[21] I. V. Mingalev, N. M. Astafieva, K. G. Orlov, V. S. Mingalev, and O. V. Mingalev, "Simulation study of the initial stage of the origin of cyclonic and anticyclonic pairs in the intertropical convergence zone," in Proceedings of the 34th Annual Seminar on Physics of Auroral Phenomena, pp. 189-192, Polar Geophysical Institute, Apatity, Russia, 2011.

[22] I. V. Mingalev, N. M. Astafieva, K. G. Orlov, V. S. Mingalev, O. V. Mingalev, and V. M. Chechetkin, "Possibility of a detection of tropical cyclones and hurricanes formation according to satellite remote sensing," in Actual Problems in Remote Sensing of the Earth from Space, vol. 8, no. 2, pp. 290-296, Space Research Institute, Moscow, Russia, 2011, in Russian.

[23] I. V. Mingalev, N. M. Astafieva, K. G. Orlov, V. M. Chechetkin, V. S. Mingalev, and O. V. Mingalev, "Numerical simulation of formation of cyclone vortex flows in the intertropical zone of convergence and their early detection," Cosmic Research, vol. 50, no. 3, pp. 233-248, 2012.

[24] V. S. Mingalev, I. V. Mingalev, O. V. Mingalev, A. M. Oparin, and K. G. Orlov, "Generalization of the hybrid monotone secondorder finite difference scheme for gas dynamics equations to the case of unstructured 3D grid," Computational Mathematics and Mathematical Physics, vol. 50, no. 5, pp. 877-889, 2010.

[25] T.-C. Chen, J.-D. Tsay, M.-C. Yen, and E. O. Cayanan, "Formation of the Philippine twin tropical cyclones during the 2008 summer monsoon onset," Weather and Forecasting, vol. 25, no. 5, pp. 1317-1341, 2010.

[26] R. N. Ferreira and W. H. Schubert, "Barotropic aspects of ITCZ breakdown," Journal of the Atmospheric Sciences, vol. 54, no. 2, pp. 261-285, 1997.

[27] C.-C. Wang and G. Magnusdottir, "ITCZ breakdown in threedimensional flows," Journal of the Atmospheric Sciences, vol. 62, no. 5, pp. 1497-1512, 2005.

[28] C.-C. Wang and G. Magnusdottir, "The ITCZ in the Central and Eastern Pacific on synoptic time scales," Monthly Weather Review, vol. 134, no. 5, pp. 1405-1421, 2006.

[29] D. S. Nolan, S. W. Powell, C. Zhang, and B. E. Mapes, "Idealized simulations of the intertropical convergence zone and its multilevel flows," Journal of the Atmospheric Sciences, vol. 67, no. 12, pp. 4028-4053, 2010.

[30] W. M. Frank and P. E. Roundy, "The role of tropical waves in tropical cyclogenesis," Monthly Weather Review, vol. 134, no. 9, pp. 2397-2417, 2006.

[31] A. J. Broccoli, K. A. Dahl, and R. J. Stouffer, "Response of the ITCZ to Northern Hemisphere cooling," Geophysical Research Letters, vol. 33, no. 1, Article ID L01702, 2006.

[32] A. Fedorov, M. Barreiro, G. Boccaletti, R. Pacanowski, and S. G. Philander, "The freshening of surface waters in high latitudes: effects on the thermohaline and wind-driven circulations," Journal of Physical Oceanography, vol. 37, no. 4, pp. 896-907, 2007.

[33] J. C. H. Chiang and A. R. Friedman, "Extratropical cooling, interhemispheric thermal gradients, and tropical climate change," Annual Review of Earth and Planetary Sciences, vol. 40, no. 1, pp. 383-412, 2012. 

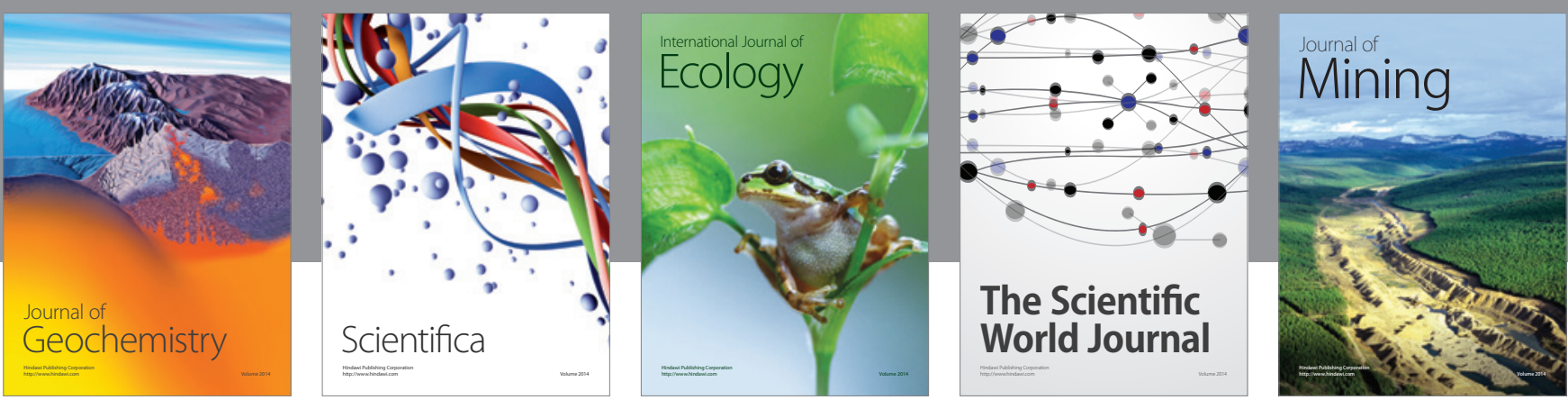

The Scientific World Journal
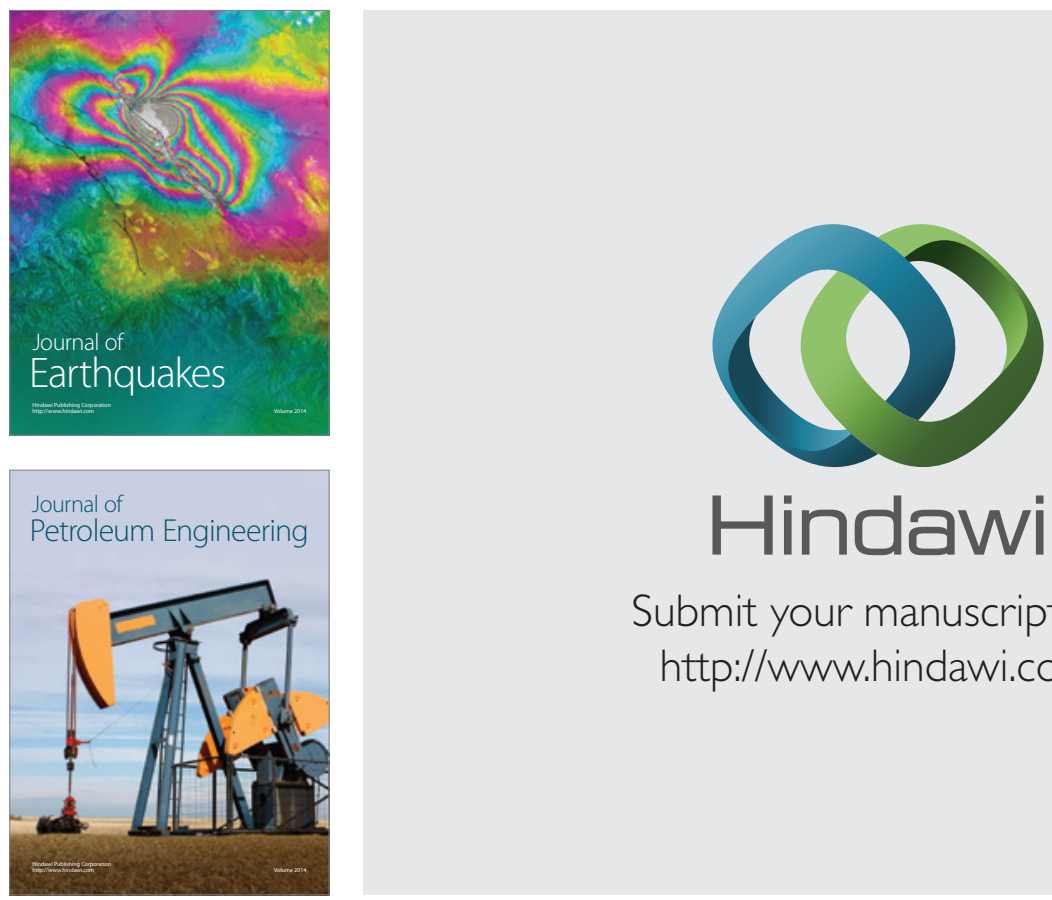

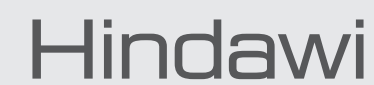

Submit your manuscripts at

http://www.hindawi.com
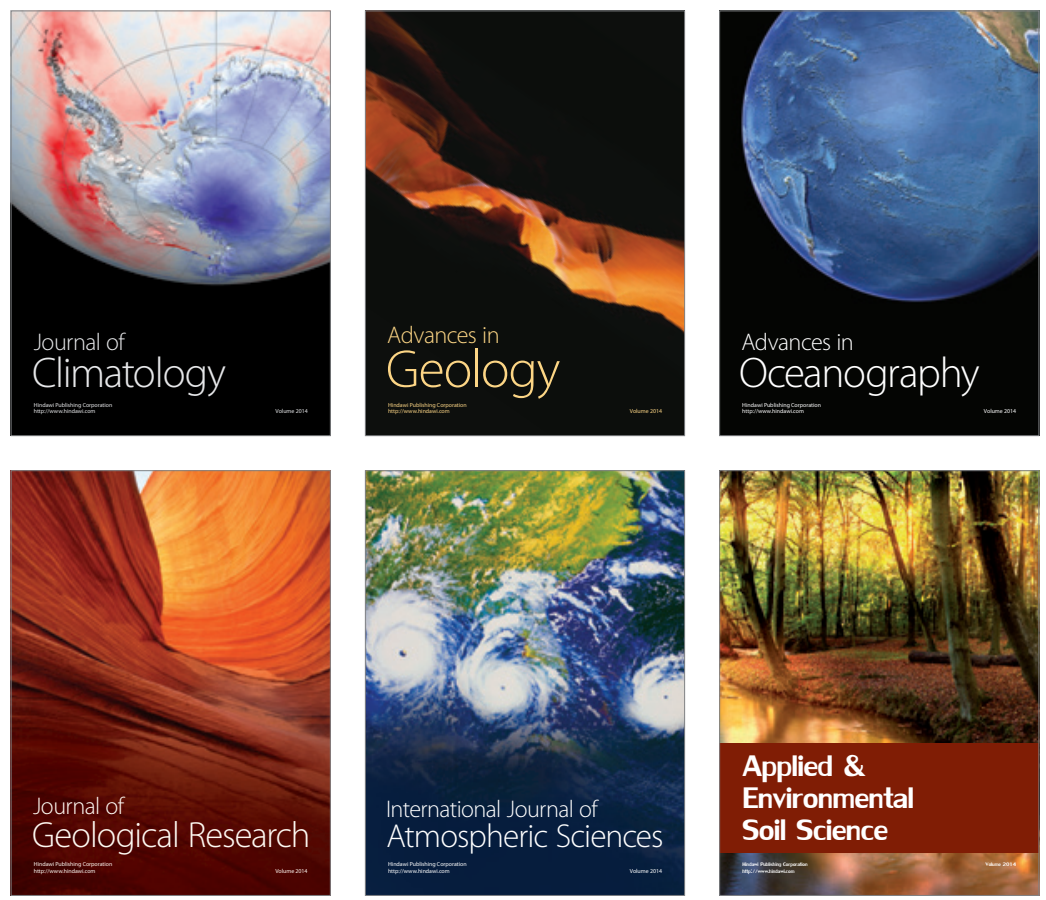
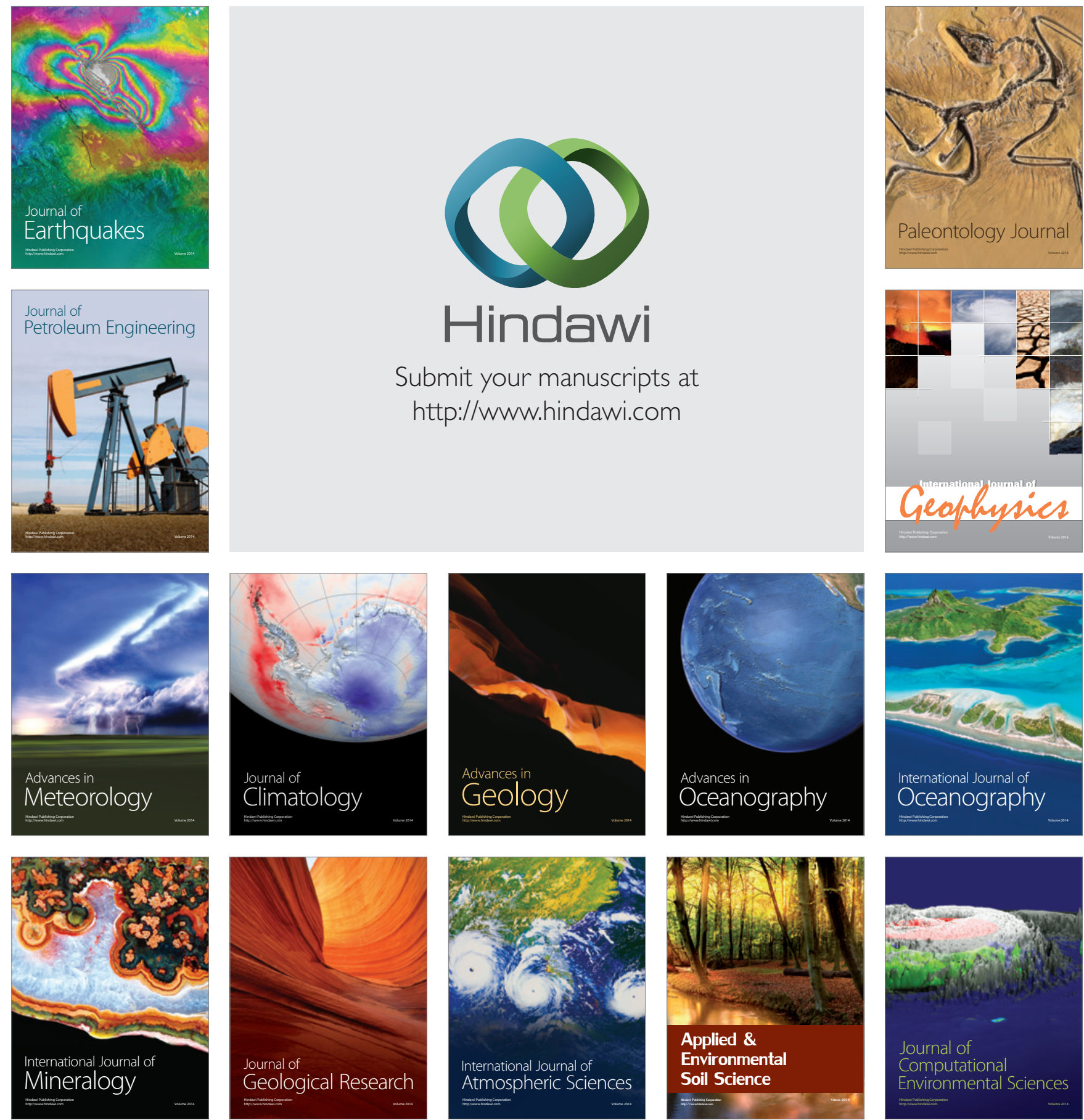